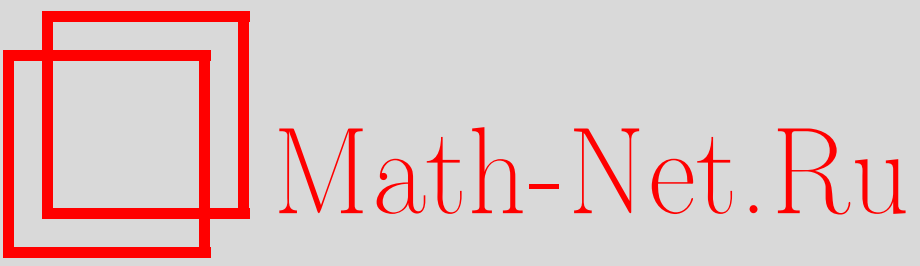

В. Г. Задорожний, М. Е. Семенов, Н. И. Сельвесюк, И. И. Ульшин, В. С. Ножкин, Статистические характеристики решений системы стохастической модели переноса, Матем. моделирование, 2020, том 32, номер 5, 21-43

DOI: https://doi.org/10.20948/mm-2020-05-02

Использование Общероссийского математического портала Math-Net.Ru подразумевает, что вы прочитали и согласны с пользовательским соглашением

http: //www.mathnet.ru/rus/agreement

Параметры загрузки:

IP : 54.224 .135 .184

26 апреля 2023 г., $17: 58: 28$ 


\title{
СТАТИСТИЧЕСКИЕ ХАРАКТЕРИСТИКИ РЕШЕНИЙ СИСТЕМЫ СТОХАСТИЧЕСКОЙ МОДЕЛИ ПЕРЕНОСА
}

\author{
(C) 2020 2. $\quad$ В.Г. Задорожний ${ }^{1,3}$, М.Е. Семенов ${ }^{1,2,3}$, Н.И. Сельвесюк \\ И.И. Ульшин ${ }^{3}$, В.С. Ножкин ${ }^{3}$
}

${ }^{1}$ Воронежский государственный университет, Воронеж

${ }^{2}$ Федеральный исследовательский центр «Единая геофизическая служба Российской академии наук» (ФИЦ ЕГС РАН), Москва

${ }^{3}$ ВУНЦ ВВС «ВВА им. проф. Н.Е. Жуковского и Ю.А. Гагарина», Воронеж

${ }^{4}$ ФГУП «Государственный научно-исследовательский институт авиационных систем», Москва

nozhkin-v@list.ru

Работа М.Е. Семенова (см. разд.3,4) выполнена при финансовой поддержке РНФ (грант 19-11-00197)

DOI: $10.20948 / \mathrm{mm}-2020-05-02$

Предлагается стохастическая модель переноса, формализуемая с помощью дифференциальных уравнений со случайными параметрами. Получены явные выражения для математического ожидания и второй моментной функции решения соответствующих уравнений. Определена оценка степени влияния случайных факторов на систему в случае замены случайного коэффициента уравнения его математическим ожиданием. Приведен пример, демонстрирующий эффективность предлагаемого подхода в случае гауссова распределения случайных коэффициентов, позволяющий определить математическое ожидание и вторую моментную функцию в рамках модельных представлений. В качестве приложения рассматривается модель переноса тепла и влаги в приземном слое атмосферы.

Ключевые слова: стохастическая модель, случайный процесс, характеристический функционал, математическое ожидание, дисперсионная функция, влияние случайных факторов.

\section{STATISTICAL CHARACTERISTICS OF SOLUTIONS OF THE SYSTEM OF THE STOCHASTIC TRANSFER MODEL}

\author{
V.G. Zadorozhniy ${ }^{1,3}$, M.E. Semenov ${ }^{1,2,3}$, N.I. Sel'vesyuk ${ }^{4}$, I.I. Ulshin ${ }^{3}$, V.S. Nozhkin ${ }^{3}$
}


${ }^{1}$ Voronezh State University, Voronezh

${ }^{2}$ Geophysical Survey of Russia Academy of Sciences, Moscow

${ }^{3}$ MESC AF «N.E. Zhukovsky and Y.A. Gagarin Air Force Academy», Voronezh

${ }^{4}$ State Research Institute of Aviation Systems, Moscow

The paper proposes a new stochastic transport model formalized in the framework of differential equations with random parameters. Explicit formulas for the mathematical expectation and the second-moment function for solving the corresponding equations are given. The estimation of the influence of random factors on the system, in the case of replacing the random coefficient of the equation by its mathematical expectation, is determined. An example with Gaussian distribution of random coefficients is also presented and discusser. The paper shows that the proposed model is applicable to the description of heat and moisture transfer processes in the surface layer of the atmosphere.

Key words: stochastic model, random process, characteristic functional, mathematical expectation, dispersion function, influence of random factors.

\section{1. Введение}

Гидродинамическое моделирование является основным методом прогнозирования полей метеорологических величин. Методы гидродинамического моделирования основаны на численном решении уравнений движения, притока тепла, неразрывности, переноса влаги и атмосферных примесей, являющихся математическими выражениями законов физики (законы сохранения импульса, энергии и массы), а также уравнения состояния [1].

Лишь несколько стран в мире развивают собственные технологии моделирования глобальной атмосферы. Из-за ограниченности вычислительных ресурсов пространственное разрешение таких моделей недостаточно для прямого полного описания некоторых процессов (например, глубокой конвекции), поэтому такие процессы возможно описать только параметрически, оперируя характеристиками атмосферы на масштабе характерного размера ячейки сетки [2]. В связи с этим чаще всего прибегают к региональным (мезомасштабным) моделям, которые, в свою очередь, также не в полной мере соответствуют реальным физическим процессам, протекающим в атмосфере. Например, региональная гидродинамическая модель COSMO$\mathrm{Ru}$, успешно применявшаяся для прогноза мезомасштабных метеорологических явлений во время сочинской олимпиады [3], имеет разрешение до 7 км [3], но и с ее помощью невозможно прогнозировать некоторые погодные явления локального характера. Это связано с тем, что многие из них имеют меньший характерный масштаб и сложную природу образования, которую в настоящее время затруднительно описать формально для полной автоматизации прогноза с приемлемым уровнем успешности. Особо отметим, что необходим учет неупорядоченных хаотических возмущений, связанных с не- 
однородностями в поле ветра, поскольку компоненты скорости ветра явным образом входят в уравнения динамики метеорологических величин. Такой учет возможен лишь на микроуровне. По этой причине целый ряд явлений погоды (например, грозы, туманы, внутримассовая облачность и др.) прогнозируются в основном специалистами-синоптиками на местах, которые хорошо знают условия их образования и развития в конкретном регионе.

Определенные погрешности в прогнозировании при помощи гидродинамических моделей вносят и начальные условия. Проблема достижения высокой точности возникла уже после первых численных экспериментов по моделированию эволюции атмосферы на долгие сроки. Еще в 60-х годах $\mathrm{XX}$ века установлено, что сколь угодно малые ошибки в начальных данных с течением времени трансформируются в весьма значительные. За пределами двухнедельного периода ошибки детализированного по дням модельного прогноза вырастают до уровня ошибок случайного прогноза [3]. Аналогичный факт был отмечен еще в знаменитой системе Лоренца [4], появившейся как результат моделирования конвективных движений в подогреваемом снизу слое жидкости.

Всемирной метеорологической организацией установлено, что повышение масштабируемости кодов моделей является одним из главных приоритетов в прогнозировании погоды и климата на ближайшие 10 лет. Поэтому вычислительная эффективность станет лимитирующим фактором номер один для моделирования погодных явлений с высоким разрешением [3]. Исходя из этого разработка микрогидродинамических моделей, позволяющих прогнозировать локальные опасные явления погоды, представляется весьма важной и актуальной.

В настоящей работе представлены результаты создания локальной гидродинамической модели переноса влаги, учитывающей неоднородности движения воздушных масс в рамках теории случайных процессов, а также приведена оценка степени влияния случайных факторов при замене случайного коэффициента уравнения его математическим ожиданием. Практическая значимость полученных результатов обусловлена тем, что подавляющее большинство опасных явлений погоды связано с повышенным влагосодержанием в атмосфере.

\section{2. Постановка задачи}

Один из общих принципов прогнозирования опасных явлений погоды, связанных с повышенным содержанием влаги в атмосфере, описан в методиках [5,6], а также в [1]. Данный принцип основан на численном решении дифференциальных уравнений переноса влаги в атмосфере. Из различных характеристик влажности (абсолютная и относительная влажность, точка 
росы и т.д.) выбрана массовая доля водяного пара $s$, так как она является наиболее консервативной характеристикой. Одновременно рассматривается и максимальная массовая доля водяного пара $s_{m}$, представляющая собой максимальное количество водяного пара в граммах в одном грамме влажного воздуха при определенных значениях давления и температуры.

Процесс изменения массовой доли описывается уравнением переноса влаги [1]

$$
\frac{d s}{d t}=-\frac{m}{\rho}+k^{\prime} \Delta s+\frac{\partial}{\partial z} k \frac{\partial s}{\partial z},
$$

где $m$ - абсолютная скорость конденсации (сублимации); $\rho$ - плотность воздуха; $k^{\prime}$ и $k$ - коэффициенты турбулентной вязкости; $\Delta-$ двумерный оператор Лапласа.

Зачастую турбулентным влагообменом пренебрегают. Тогда в условиях постоянства влагосодержания в рассматриваемой области уравнение (1) упрощается, и рассматриваются два случая.

Первый случай имеет место, когда температура точки росы $T_{d}$ меньше температуры воздуха $T_{b}$, а массовая доля водяного пара меньше максимальной массовой доли. Тогда массовая доля в движущейся частице остается неизменной, и уравнение переноса влаги принимает вид

$$
\frac{\partial s}{\partial t}+u \frac{\partial s}{\partial x}+v \frac{\partial s}{\partial y}+\widehat{w} \frac{\partial s}{\partial p}=0
$$

где $u, v$ - проекции вектора скорости на соответствующие оси координат; $\widehat{w}$ - аналог вертикальной скорости в $p$-системе координат [1].

Второй случай наблюдается, когда температура точки росы равна температуре воздуха, а массовая доля равна максимальной массовой доле. В этом случае происходит конденсация (сублимация) и уравнение переноса влаги с учетом уравнений притока тепла и Клапейрона-Клаузиуса принимает вид

$$
\frac{\partial s_{m}}{\partial t}+u \frac{\partial s_{m}}{\partial x}+v \frac{\partial s_{m}}{\partial y}+\widehat{w} \frac{\partial s_{m}}{\partial p}=F\left(T_{b}, p\right) \widehat{w}
$$

где $F\left(T_{b}, p\right)$ - функция давления и температуры, определяемая соотношением

$$
F\left(T_{b}, p\right)=\frac{\frac{s_{m}}{p}\left(\frac{R}{c_{p}}-\frac{Q}{c_{p} T_{b}} \frac{R}{R_{\Pi}}\right)}{1+\frac{s_{m} Q\left(Q-R_{\Pi} T_{b}\right)}{c_{p} R_{\Pi} T_{b}^{2}}} .
$$


Здесь $Q$ - скрытая теплота конденсации (сублимации); $R_{\Pi}$ - удельная газовая постоянная водяного пара; $R$ - удельная газовая постоянная сухого воздуха; $c_{p}$ - удельная теплоемкость воздуха при постоянном давлении.

Поскольку $s_{m}$ является функцией температуры, необходимо одновременно прогнозировать и температуру воздуха, используя уравнение притока тепла в виде

$$
\frac{\partial T_{b}}{\partial t}+u \frac{\partial T_{b}}{\partial x}+v \frac{\partial T_{b}}{\partial y}=\frac{\left(\gamma_{a}-\gamma\right)}{g \rho} \widehat{w},
$$

где $\gamma_{a}$ - сухоадиабатический градиент температуры; $\gamma$ - вертикальный градиент температуры; $g$ - ускорение свободного падения.

Традиционно прогноз влажности осуществляется следующим образом. На основании имеющихся данных о фактической влажности и температуре воздуха определяется достижение состояния насыщения водяного пара. В рамках стандартной схемы гидродинамического моделирования на каждом шаге по времени получают прогноз значений проекций вектора скорости, температуры воздуха и вертикальной скорости в $p$-системе координат [1]. Если состояние насыщения водяного пара не достигнуто, то для расчета изменения массовой доли водяного пара используется стандартное уравнение переноса, в котором компоненты вектора скорости трактуются как усредненные прогностические величины, в противном случае по достижению состояния насыщения рассчитывается функция давления и температуры $F\left(T_{b}, p\right)$, а затем скорость конденсации. Далее делается следующий шаг по времени, который начинается с проверки на состояние насыщения.

Такой подход представляется вполне оправданным при моделировании крупно- и среднемасштабных процессов, имеющих характерный пространственный масштаб, превышающий десятки километров, когда используются усредненные значения метеорологических величин. Однако, как было отмечено выше, некоторые явления погоды могут иметь характерный пространственный масштаб, не превышающий нескольких километров. Поэтому традиционный подход в этом случае становится неприемлемым.

В настоящей работе предлагается альтернативный способ описания динамики влагосодержания и температуры малых пространственно временных масштабов, основанный на трактовке компонент скорости ветра в уравнениях (2)-(4) как случайных процессов.

В качестве обоснования такого подхода к прогнозированию переноса влаги в атмосфере, позволяющего на модельном уровне учитывать турбу- 
лентные свойства атмосферы, проведена серия экспериментов, связанных с регистрацией изменения параметров ветра на относительно небольших временных интервалах. Проведены расчеты по полученным данным проекций вектора скорости и усреднения на различных интервалах времени. На рис.1 приведены примеры графиков изменения значений проекции вектора скорости при интервалах усреднения 15, 60 и 180 с. (на рисунке представлены результаты измерений времени в течение 21 минуты).

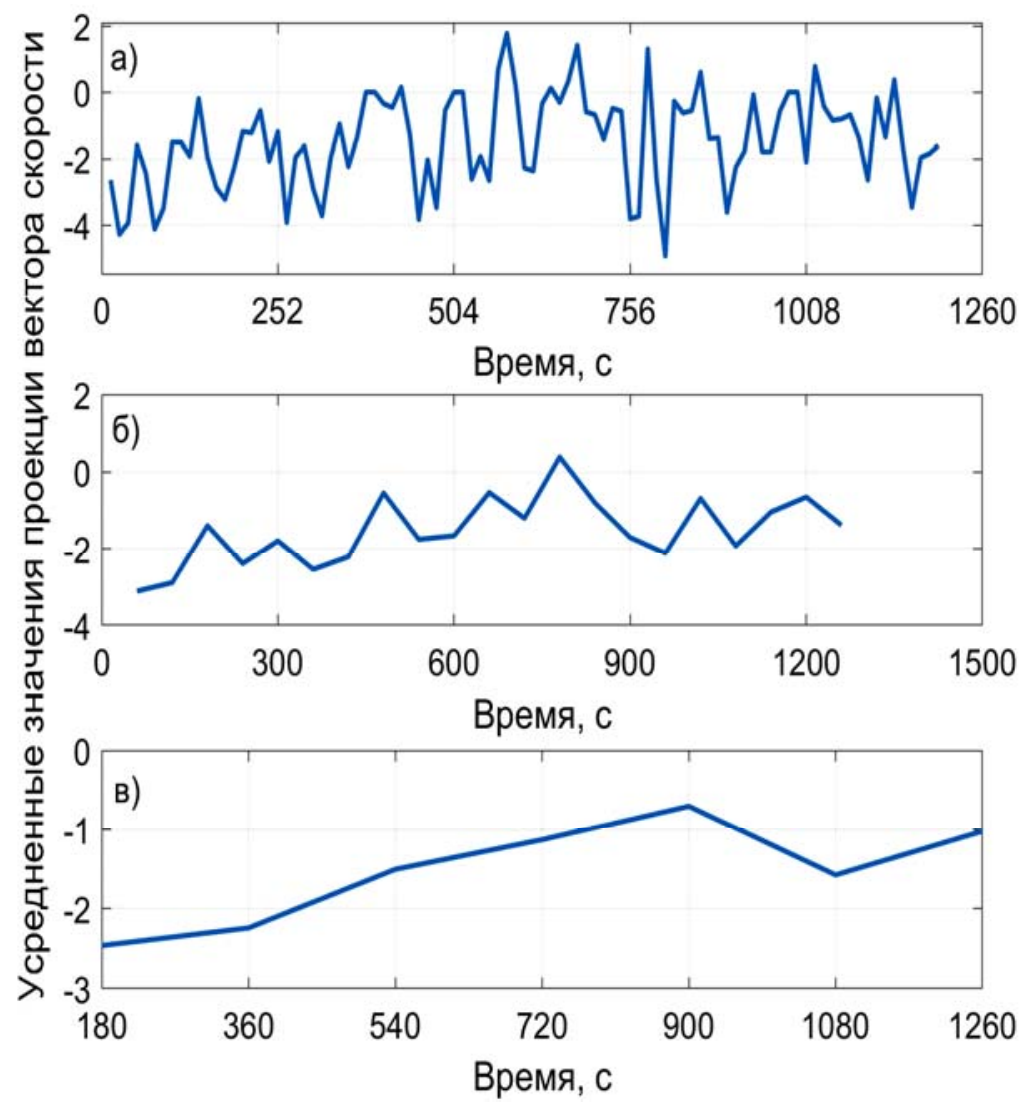

Рис.1. а) усредненные значения проекции вектора скорости за 15 секунд;

б) усредненные значения проекции вектора скорости за 60 секунд;

в) усредненные значения проекции вектора скорости за 180 секунд.

Из приведенных рисунков однозначно следует, что при рассмотрении процессов, имеющих характерный временной масштаб порядка нескольких минут, компоненты вектора скорости вполне допускают трактовку в терминах случайных процессов.

Одной из важных характеристик случайных процессов является автокорреляционная функция $\Phi(k)$, показывающая зависимость взаимосвязи между функцией и ее сдвинутой копией от величины временного сдвига $k$. Анализ автокорреляционной функции делает возможным нахождение повторяющихся закономерностей, таких как наличие периодического сигнала, скрытого шумом, или определение отсутствующей основной частоты в сигнале, представленном широким спектром гармонических частот. 
На рис.2 представлен график автокорреляционной функции, построенной по наблюдениям за горизонтальной компонентой скорости ветра.

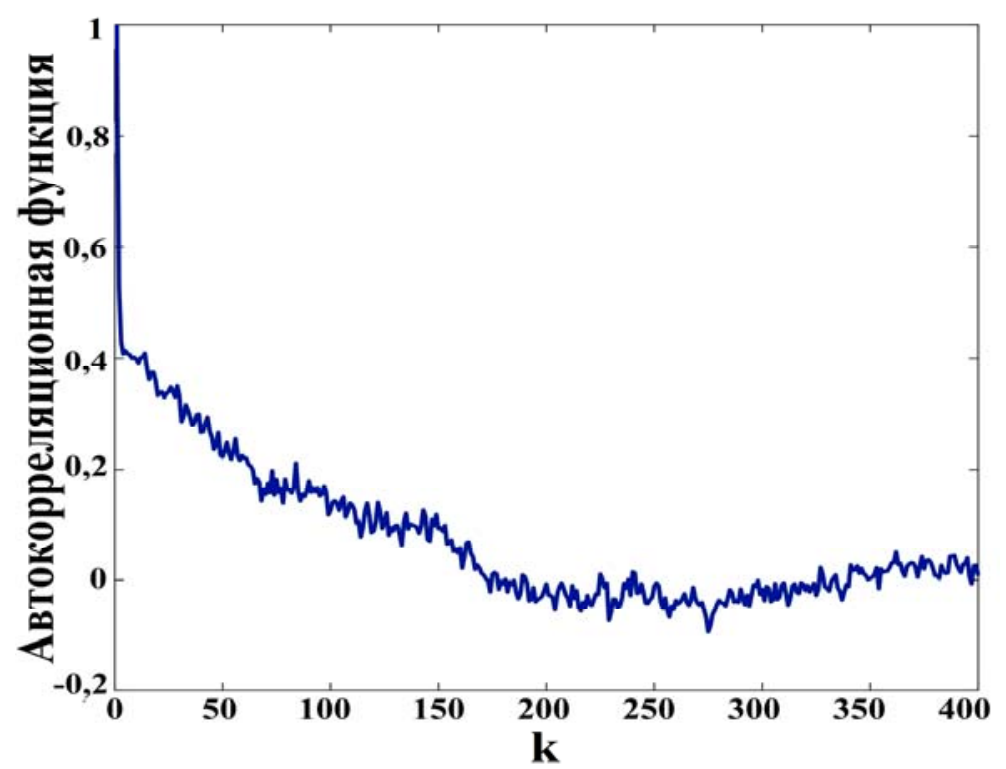

Рис.2. Автокорреляционная функция $\Phi(k)$ проекции вектора скорости.

Эксперимент показал, что в условиях малоградиентного барического поля, когда создаются благоприятные условия для образования туманов и густых дымок, автокорреляционная функция, полученная по наблюдениям в дневные часы, имеет степенной характер убывания. Этот факт в дальнейшем использовался в ходе практической реализации предложенного подхода.

Для упрощения задачи моделирования процессов тепло- и влагообмена в атмосфере ось $x$ была ориентирована по направлению преимущественного переноса воздушной массы, во внимание не принимались вертикальные движения воздуха, давление $p=p(x)$ считалось не зависящей от времени функцией координат и вводились следующие обозначения $F\left(T_{B}, p\right) \widehat{w}(x)=$ $=f\left(x, T_{B}\right) ; f_{1}(x)=\frac{\left(\gamma_{a}-\gamma\right)}{g \rho} \widehat{w}(x)$. Данные функции в дальнейшем считались детерминированными. Таким образом, система уравнений (2)-(4) была преобразована к виду

$$
\begin{aligned}
& \frac{\partial s}{\partial t}+\varepsilon(t) \frac{\partial s}{\partial x}=0, \quad T_{d}<T_{b}, \quad s<s_{m} ; \\
& \frac{\partial s_{m}}{\partial t}+\varepsilon(t) \frac{\partial s_{m}}{\partial x}=f\left(x, T_{B}\right), \quad T_{d}=T_{b}, \quad s=s_{m} ; \\
& \frac{\partial T_{b}}{\partial t}+\varepsilon(t) \frac{\partial T_{B}}{\partial x}=f_{1}(x),
\end{aligned}
$$


с детерминированными начальными условиями:

$$
s(0, x)=s_{0}(x) ; s_{m}(0, x)=s_{m 0}(x) ; T_{B}(0, x)=T_{B 0}(x) ;-\infty<x<\infty,
$$

где $\varepsilon(t)$ - случайный процесс, характеристики которого идентифицируются на основе пространственно-временного распределения скорости ветра.

Основная цель настоящей работы состоит в нахождении математического ожидания и дисперсионной функции решения системы уравнений (5)-(7) с начальными условиями (8). Решение указанной задачи осуществляется путем перехода к детерминированному уравнению на основе метода, предложенного в [7-9] и связанного с понятием вариационной производной. Первым этапом в решении указанной выше системы является решение уравнения (7), формально не зависящего от других уравнений.

\section{3. Переход к детерминированной задаче}

Случайный процесс $\varepsilon(t)$ считаем заданным характеристическим функционалом [8]

$$
\varphi(v)=M\left[\exp \left(i \int_{T} \varepsilon(\tau) v(\tau) d \tau\right)\right]
$$

где функция $v$ принадлежит пространству $L_{1}(T)$ суммируемых на отрезке $T$ функций с нормой $\|v\|=\int_{T}|v(\tau)| d \tau ; M$ - математическое ожидание по функции распределения процесса $\varepsilon(t) ; T$ - отрезок времени, на котором изучается процесс.

Умножим уравнение (7) на $\exp \left(i \int_{T} \varepsilon(\tau) v(\tau) d \tau\right)$ и применим операцию математического ожидания по функции распределения случайного процесса $\varepsilon(t)$ к полученному равенству

$$
\begin{aligned}
& M\left[\frac{\partial T_{B}}{\partial t} \exp \left(i \int_{T} \varepsilon(\tau) v(\tau) d \tau\right)\right]=M\left[-\varepsilon(t) \frac{\partial T_{B}}{\partial x} \exp \left(i \int_{T} \varepsilon(\tau) v(\tau) d \tau\right)\right]+ \\
& +f_{1}(x) M\left[\underset{T}{\left.\exp \left(i \int_{T} \varepsilon(\tau) v(\tau) d \tau\right)\right]}\right.
\end{aligned}
$$

Для дальнейших построений введем вспомогательное отображение:

$$
y(t, x, v)=M\left[T_{B}(t, x) \exp \left(i \int_{T} \varepsilon(\tau) v(\tau) d \tau\right)\right],
$$


где $x \in R ; v(t) \in L_{1}(T)$. Тогда уравнение (10) можно представить в виде

$$
\frac{\partial y(t, x, v)}{\partial t}=i \frac{\delta}{\delta v(t)} \frac{\partial}{\partial x} y(t, x, v)+f_{1}(x) \varphi(v)
$$

с начальным условием

$$
y(0, x, v)=T_{B 0}(x) \varphi(v),
$$

где $\delta / \delta v(t)$ - вариационная производная. Детальное описание операции вариационного дифференцирования приведено, например, в [7-11].

Непосредственно из определений следует, что математическое ожидание решения уравнения (7) с начальным условием (8) может быть получено из соотношения (11), в котором функцию $v$ следует положить равной нулю. Иными словами имеет место равенство

$$
y(t, x, 0)=M\left[T_{B}(t, x)\right] .
$$

Таким образом, для нахождения математического ожидания решения уравнения (7) с начальным условием (8) достаточно найти решение уравнения с вариационной и обычной производными (12) при условии (13) в некоторой окрестности нулевой функции пространства $L_{1}(T)$.

\section{4. Математическое ожидание решения уравнения притока тепла}

Применив к обеим частям выражений (12) и (13) преобразование Фурье [12,13], получим

$$
\begin{aligned}
& \frac{\partial}{\partial t} F_{x}[y(t, x, v)](\xi)=i \frac{\delta}{\delta v(t)}(-i \xi) F_{x}[y(t, x, v)](\xi)+F_{x}\left[f_{1}(x)\right](\xi) \varphi(v), \\
& F_{x}[y(0, x, v)](\xi)=F_{x}\left[T_{B 0}(x)\right](\xi) \varphi(v),
\end{aligned}
$$

где $F_{x}$ - преобразование Фурье по переменной $x$, а $\xi$ - двойственная к $x$ переменная. Обозначим

$$
Y(t, \xi, v)=F_{x}[y(t, x, v)](\xi) ; b(\xi, v)=F_{x}\left[f_{1}(x)\right](\xi) \varphi(v) .
$$

Тогда соотношения (15) и (16) перепишутся в виде

$$
\begin{aligned}
& \frac{\partial}{\partial t} Y(t, \xi, v)=\xi \frac{\delta}{\delta v(t)} Y(t, \xi, v)+b(\xi, v) . \\
& Y_{0}(0, \xi, v)=F_{x}\left[T_{B 0}(x)\right](\xi) \varphi(v) .
\end{aligned}
$$

Введем вспомогательную функцию, зависящую от трех переменных $\chi(\tau, t, w)$ следующим образом $\chi(\tau, t, w)=\operatorname{sign}(w-\tau)$ при $w$, принадлежащем 
отрезку с концами $\tau$ и $t$, и $\chi(\tau, t, w)=0$ в противном случае. Дальнейшие построения опираются на следующую лемму [8]:

Лемма 1. Пусть а $(t)$ - непрерывная функиия на отрезке $T$ и функиионал $y: L_{1}(T) \rightarrow \mathbb{C}$ имеет вариационную производную $\delta y(v) / \delta v(t)$. Тогда почти при всех $t \in T$ существует производная $\frac{\partial y(v+a \chi(\tau, t))}{\partial t}$ и выполняется равенство

$$
\frac{\partial y(v+a \chi(\tau, t))}{\partial t}=a(t) \frac{\delta y(v+a \chi(\tau, t))}{\delta v(t)} .
$$

Теорема 1. Если существуют вариационные производные $\delta \varphi(v) / \delta v(t)$, $\delta b(v) / \delta v(t)$, то отображение

$$
Y(t, \xi, v)=Y_{0}(0, \xi, v+\xi \chi(0, t))+\int_{0}^{t} b(v+\xi \chi(\tau, t)) d \tau
$$

является решением задачи (17) и (18).

Доказательство. Непосредственной подстановкой (19) в (17) и (18) и использованием леммы 1 убеждаемся в справедливости теоремы.

Пусть $\stackrel{x}{*}$ - знак свертки функций по переменной $x ; F_{\xi}^{-1}$ - обратное преобразование Фурье по $\xi$.

Теорема 2. В условиях теоремы 1 отображение

$$
\begin{aligned}
& y(t, x, v)=T_{B 0}(x)^{*} F_{\xi}^{-1}[\varphi(v+\xi \chi(0, t))](x)+ \\
& +f_{1}(x)^{*} F_{\xi}^{-1}\left[\int_{0}^{t} \varphi(v+\xi \chi(\tau, t)) d \tau\right](x)
\end{aligned}
$$

является решением задачи (12), (13).

Доказательство. Применим обратное преобразование Фурье [12,13,15] к (19), получим (20).

Теорема 3. Пусть выполняются условия предыдущей теоремь, тогда

$$
M\left[T_{B}(t, x)\right]=T_{B 0}(x)^{*} F_{\xi}^{-1}[\varphi(\xi \chi(0, t))](x)+f_{1}(x)^{*} F_{\xi}^{-1}\left[\int_{0}^{t} \varphi(\xi \chi(\tau, t)) d \tau\right](x)
$$

является математическим ожиданием решения уравнения (7), при начальном условии (8).

Доказательство. Для доказательства соотношения (21) достаточно в соотношении (20) положить $v=0$. 


\section{5. Вторая моментная функция решения уравнения притока тепла}

Для определения второй моментной функции решения задачи (7) введем в рассмотрение отображение

$$
z\left(t, x, t_{1}, x_{1}, v\right)=M\left[T_{B}(t, x) T_{B}\left(t_{1}, x_{1}\right) \exp \left(i \int_{T} \varepsilon(\tau) v(\tau) d \tau\right)\right] .
$$

Отметим, что отображение $z\left(t, x, t_{1}, x_{1}, v\right)$ симметрично по парам переменных $t, x$ и $t_{1}, x_{1}$. Умножим уравнения (7), (8) на $T_{B}\left(t_{1}, x_{1}\right) \exp \left(i \int_{T} \varepsilon(\tau) v(\tau) d \tau\right)$ и применим операцию математического ожидания по функции распределения случайного процесса $\varepsilon(t)$. Получим

$$
\begin{aligned}
& M\left[\frac{\partial T_{B}}{\partial t} T_{b}\left(t_{1}, x_{1}\right) \exp \left(i \int_{T} \varepsilon(\tau) v(\tau) d \tau\right)\right]= \\
& =M\left[-\varepsilon(t) \frac{\partial T_{B}}{\partial x} T_{B}\left(t_{1}, x_{1}\right) \underset{T}{\left.\exp \left(i \int_{T} \varepsilon(\tau) v(\tau) d \tau\right)\right]+}\right. \\
& +f_{1}(x) M\left[T_{B}\left(t_{1}, x_{1}\right) \exp \left(i \int_{T} \varepsilon(\tau) v(\tau) d \tau\right)\right], \\
& M\left[T_{B}(0, x) T_{B}\left(t_{1}, x_{1}\right) \exp \left(i \int_{T} \varepsilon(\tau) v(\tau) d \tau\right)\right]= \\
& =M\left[T_{B 0}(x) T_{B}\left(t_{1}, x_{1}\right) \exp \left(i \int_{T} \varepsilon(\tau) v(\tau) d \tau\right)\right] .
\end{aligned}
$$

Используя вспомогательное отображение $z\left(t, x, t_{1}, x_{1}, v\right)$, соотношения (23), (24) запишем в виде

$$
\begin{aligned}
& \frac{\partial}{\partial t} z\left(t, x, t_{1}, x_{1}, v\right)=i \frac{\delta}{\delta v(t)} \frac{\partial}{\partial x} z\left(t, x, t_{1}, x_{1}, v\right)+f_{1}(x) y\left(t_{1}, x_{1}, v\right), \\
& z\left(0, x, t_{1}, x_{1}, v\right)=T_{B 0}(x) y\left(t_{1}, x_{1}, v\right) .
\end{aligned}
$$

Определение 1. Второй моментной функиией решения задачи (7) $c$ детерминированным начальным условием (8) называется $z\left(t, x, t_{1}, x_{1}, 0\right)$, где $z\left(t, x, t_{1}, x_{1}, v\right)$ - симметричное по парам переменных $t, x$ и $t_{1}, x_{1}$ решение задачи (25), (26) и обозначается через $M\left[T_{b}(t, x) T_{b}\left(t_{1}, x_{1}\right)\right]$. 
Теорема 4. В условиях теоремы 2 решение задачи (25), (26) имеет вид

$$
\begin{aligned}
& z\left(t, x, t_{1}, x_{1}, v\right)=T_{B 0}(x)^{*} F_{\xi}^{-1}\left[y\left(t_{1}, x_{1}, v+\xi \chi(0, t)\right)\right](x)+ \\
& +f_{1}(x)^{*} F_{\xi}^{-1}\left[\int_{0}^{t} y\left(t_{1}, x_{1}, v+\xi \chi(\tau, t)\right) d \tau\right](x),
\end{aligned}
$$

где $y\left(t_{1}, x_{1}, v\right)$ определяется соотношением (20).

Доказательство. Задача (25), (26) совпадает с точностью до обозначений с задачей (12), (13). Используя формулу (19), получаем (27).

Воспользовавшись равенством (20) и подставив его в (27), получим решение, представленное с использованием начального условия и характеристического функционала $\varphi$

$$
\begin{aligned}
& z\left(t, x, t_{1}, x_{1}, v\right)=T_{B 0}(x)^{*} F_{\xi}^{-1}\left[T _ { B 0 } ( x _ { 1 } ) ^ { * } F _ { \xi _ { 1 } } ^ { - 1 } \left[\varphi \left(v+\xi_{1} \chi\left(0, t_{1}\right)+\right.\right.\right. \\
& \left.+\xi \chi(0, t))]\left(x_{1}\right)+f_{1}\left(x_{1}\right)^{*} F_{\xi_{1}}^{-1}\left[\int_{0}^{t_{1}} \varphi\left(v+\xi_{1} \chi\left(\tau, t_{1}\right)+\xi \chi(0, t)\right) d \tau\right]\left(x_{1}\right)\right](x)+ \\
& +f_{1}(x)^{*} F_{\xi}^{-1}\left[\int _ { 0 } ^ { t } \left(T_{B 0}\left(x_{1}\right)^{*} F_{\xi_{1}}^{-1}\left[\varphi\left(v+\xi_{1} \chi\left(0, t_{1}\right)+\xi \chi(\eta, t)\right)\right]\left(x_{1}\right)+\right.\right. \\
& \left.\left.+f_{1}\left(x_{1}\right)^{*} F_{\xi_{1}}^{-1}\left[\int_{0}^{t_{1}} \varphi\left(v+\xi_{1} \chi\left(\tau, t_{1}\right)+\xi \chi(\eta, t)\right) d \tau\right]\left(x_{1}\right)\right) d \eta\right](x) .
\end{aligned}
$$

Теорема 5. В условиях предыдущей теоремы вторая моментная функизи решения задачи (7), (8) имеет вид

$$
\begin{aligned}
& M\left[T_{\theta}(t, x) T_{\theta}\left(t_{1}, x_{1}\right)\right]=T_{B 0}(x)^{*} F_{\xi}^{-1}\left[T _ { B 0 } ( x _ { 1 } ) ^ { * } F _ { \xi _ { 1 } } ^ { - 1 } \left[\varphi \left(\xi_{1} \chi\left(0, t_{1}\right)+\right.\right.\right. \\
& \left.+\xi \chi(0, t))]\left(x_{1}\right)+f_{1}\left(x_{1}\right)^{x_{1}} F_{\xi_{1}}^{-1}\left[\int_{0}^{t_{1}} \varphi\left(\xi_{1} \chi\left(\tau, t_{1}\right)+\xi \chi(0, t)\right) d \tau\right]\left(x_{1}\right)\right](x)+ \\
& +f_{1}(x)^{*} F_{\xi}^{-1}\left[\int _ { 0 } ^ { t } \left[T_{\theta 0}\left(x_{1}\right)^{*} F_{\xi_{1}}^{-1}\left[\varphi\left(\xi_{1} \chi\left(0, t_{1}\right)+\xi \chi(\eta, t)\right)\right]\left(x_{1}\right)+\right.\right. \\
& \left.\left.+f_{1}\left(x_{1}\right)^{*} F_{\xi_{1}}^{-1}\left[\int_{0}^{t_{1}} \varphi\left(\xi_{1} \chi\left(\tau, t_{1}\right)+\xi \chi(\eta, t)\right) d \tau\right]\left(x_{1}\right)\right) d \eta\right](x) .
\end{aligned}
$$


Доказательство. Поскольку $z\left(t, x, t_{1}, x_{1}, 0\right)=M\left[T_{b}(t, x) T_{b}\left(t_{1}, x_{1}\right)\right]$, то из (28) при $v=0$ получаем (29).

Важной статистической характеристикой случайного процесса является дисперсионная функция $D\left[T_{b}(t, x)\right]=M\left[T_{b}^{2}(t, x)\right]-\left(M\left[T_{b}(t, x)\right]\right)^{2}$.

Теорема 6. Пусть выполняются условия теоремь 5, тогда дисперсионная функция решения задачи (7), (8) имеет вид

$$
\begin{aligned}
& D\left[T_{B}(t, x)\right]=T_{B 0}(x)^{*} F_{\xi}^{-1}\left[T_{B 0}(x)^{*} F_{\xi}^{-1}[\varphi(2 \xi \chi(0, t)](x)+\right. \\
& \left.+f_{1}(x)^{*} F_{\xi}^{-1}\left[\int_{0}^{t} \varphi(\xi \chi(\tau, t)+\xi \chi(0, t)) d \tau\right](x)\right](x)+ \\
& +f_{1}(x)^{*} F_{\xi}^{-1}\left[\int _ { 0 } ^ { t } \left(T_{B 0}(x)^{*} F_{\xi}^{-1}\left[\varphi\left(\xi \chi(0, t)+\xi \chi\left(\tau_{1}, t\right)\right)\right](x)+\right.\right. \\
& \left.\left.+f_{1}(x)^{*} F_{\xi}^{-1}\left[\int_{0}^{t} \varphi\left(\xi \chi(\tau, t)+\xi \chi\left(\tau_{1}, t\right)\right) d \tau\right](x)\right] d \tau_{1}\right](x)- \\
& \quad \begin{array}{c}
x \\
\left.T_{B 0}(x)^{*} F_{\xi}^{-1}[\varphi(\xi \chi(0, t))](x)+f_{1}(x)^{*} F_{\xi}^{-1}\left[\int_{0}^{t} \varphi(\xi \chi(\tau, t)) d \tau\right](x)\right)^{2} .
\end{array}
\end{aligned}
$$

Доказательство. Подставив в формулу определения дисперсионной функции равенства (21) и (29) и положив в последнем выражении $t_{1}=t$, $x_{1}=x$, получим (30).

\section{6. Уравнение притока тепла с гауссовым случайным процессом}

Идентификация характеристического функционала случайного процес$\mathrm{ca}$, соответствующего горизонтальной компоненте скорости ветра - отдельная непростая задача. В [14] на основе экспериментальных наблюдений установлено, что наблюдаемые реализации не отвергают гипотезу о нормальном распределении горизонтальной компоненты скорости ветра не менее, чем в $82 \%$ случаев. Поэтому отдельное рассмотрение случая, соответствующего гауссову случайному процессу, представляется целесообразным. Характеристический функционал гауссова случайного процесса имеет вид [9]

$$
\varphi(v)=\exp \left(i \int_{T} M[\varepsilon(\tau)] v(\tau) d \tau-\frac{1}{2} \int_{T T} \int_{T} b\left(\tau_{1}, \tau_{2}\right) v\left(\tau_{1}\right) v\left(\tau_{2}\right) d \tau_{1} d \tau_{2}\right),
$$


где $b\left(\tau_{1}, \tau_{2}\right)=M\left[\varepsilon\left(\tau_{1}\right) \varepsilon\left(\tau_{2}\right)\right]-M\left[\varepsilon\left(\tau_{1}\right)\right] M\left[\varepsilon\left(\tau_{2}\right)\right] \quad-\quad$ ковариационная функция процесса $\varepsilon(t)$.

Для упрощения дальнейших громоздких выкладок введем обозначения

$$
A(a, b)=\int_{a}^{b} M[\varepsilon(\tau)] d \tau ; B(a, b, c, d)=\int_{a c}^{b} \int_{c}^{d} b\left(\tau_{1}, \tau_{2}\right) d \tau_{1} d \tau_{2},
$$

а также матрицу и векторы

$$
G(a, b, c, d)=\left(\begin{array}{ll}
B(a, b, a, b) & B(a, b, c, d) \\
B(a, b, c, d) & B(c, d, c, d)
\end{array}\right), \overrightarrow{\mathbf{x}}=\left(\begin{array}{c}
x \\
x_{1}
\end{array}\right), \vec{\xi}=\left(\begin{array}{c}
\xi \\
\xi_{1}
\end{array}\right)
$$

ниже и далее $(\cdot, \cdot)$ - обозначается скалярное произведение в евклидовом пространстве.

Теорема 7. Пусть $\varepsilon(t)$ - гауссов случайный процесс, тогда математическое ожидание $M\left[T_{B}(t, x)\right]$ решения задачи $(7),(8)$ имеет вид

$$
\begin{aligned}
& M\left[T_{B}(t, x)\right]=T_{B 0}(x)^{*} \frac{1}{\sqrt{2 \pi B(0, t, 0, t)}} \exp \left(-\frac{(x-A(0, t))^{2}}{2 B(0, t, 0, t)}\right)+ \\
& +f_{1}(x)^{*} \int_{0}^{x} \frac{1}{\sqrt{2 \pi B(\tau, t, \tau, t)}} \exp \left(-\frac{(x-A(\tau, t))^{2}}{2 B(\tau, t, \tau, t)}\right) d \tau .
\end{aligned}
$$

Доказательство. Подставив характеристический функционал (31) в соотношение (21) и воспользовавшись определением функции $\chi$ и свойствами преобразования Фурье, получим (32).

Теорема 8. Пусть выполняются условия предыдущей теоремы, тогда вторая моментная функция имеет вид

$$
\begin{aligned}
& M\left[T_{b}(t, x) T_{b}\left(t_{1}, x_{1}\right)\right]= \\
& ={\frac{T_{b 0}(x) T_{B 0}\left(x_{1}\right)}{\sqrt{\operatorname{det} G\left(0, t, 0, t_{1}\right)}}}^{\overrightarrow{\mathbf{x}}} \exp \left[-\frac{1}{8} G^{-1}\left(0, t, 0, t_{1}\right)\left(\begin{array}{c}
x-A(0, t) \\
x_{1}-A\left(0, t_{1}\right)
\end{array}\right),\left(\begin{array}{c}
x-A(0, t) \\
x_{1}-A\left(0, t_{1}\right)
\end{array}\right)\right]+ \\
& +\int_{0}^{t_{1}}{\frac{T_{B 0}(x) f_{1}\left(x_{1}\right)}{\sqrt{\operatorname{det} G\left(\tau, t_{1}, 0, t\right)}}}^{\overrightarrow{\mathbf{x}}} \exp \left[-\frac{1}{8} G^{-1}\left(\tau, t_{1}, 0, t\right)\left(\begin{array}{c}
x-A(0, t) \\
x_{1}-A\left(\tau, t_{1}\right)
\end{array}\right),\left(\begin{array}{c}
x-A(0, t) \\
x_{1}-A\left(\tau, t_{1}\right)
\end{array}\right)\right]+ \\
& +\int_{0}^{t} \frac{T_{B 0}\left(x_{1}\right) f_{1}(x)}{{\sqrt{\operatorname{det} G\left(0, t_{1}, \eta, t\right)}}^{\overrightarrow{\mathbf{x}}}} * \exp \left[-\frac{1}{8} G^{-1}\left(0, t_{1}, \eta, t\right)\left(\begin{array}{c}
x-A\left(0, t_{1}\right) \\
x_{1}-A(\eta, t)
\end{array}\right),\left(\begin{array}{c}
x-A\left(0, t_{1}\right) \\
x_{1}-A(\eta, t)
\end{array}\right)\right]+
\end{aligned}
$$


Статистические характеристики решений системы стохастической модели ...

$$
+\int_{0}^{t} d \xi \int_{0}^{t_{1}}{\frac{f_{1}\left(x_{1}\right) f_{1}(x)}{\sqrt{\operatorname{det} G\left(\xi, t_{1}, \eta, t\right)}}}^{\stackrel{\overrightarrow{\mathbf{x}}}{*}} \exp \left[-\frac{1}{8} G^{-1}\left(\xi, t_{1}, \eta, t\right)\left(\begin{array}{c}
x-A\left(\xi, t_{1}\right) \\
x_{1}-A(\eta, t)
\end{array}\right),\left(\begin{array}{c}
x-A\left(\xi, t_{1}\right) \\
x_{1}-A(\eta, t)
\end{array}\right)\right] .
$$

Доказательство. Подставив характеристический функционал (31) в равенство (29), получим

$$
\begin{aligned}
& M\left[T_{B}(t, x) T_{B}\left(t_{1}, x_{1}\right)\right]= \\
& =T_{B 0}(x)^{*} F_{\xi}^{-1}\left[T _ { B 0 } ( x _ { 1 } ) ^ { * } F _ { \xi _ { 1 } } ^ { - 1 } \left[\operatorname { e x p } \left[i \int_{T} M[\varepsilon(\tau)]\left(\xi_{1} \chi\left(0, t_{1}, \tau\right)+\xi \chi(0, t, \tau)\right) d \tau-\right.\right.\right. \\
& -\frac{1}{2} \int_{T T} \int_{T} b\left(\tau_{1}, \tau_{2}\right)\left(\xi_{1} \chi\left(0, t_{1}, \tau_{1}\right)+\xi \chi\left(0, t, \tau_{1}\right)\right)\left(\xi_{1} \chi\left(0, t_{1}, \tau_{2}\right)+\right. \\
& \left.\left.\left.+\xi \chi\left(0, t, \tau_{2}\right)\right) d \tau_{1} d \tau_{2}\right]\right]\left(x_{1}\right)+f_{1}\left(x_{1}\right)^{x_{1}} F_{\xi_{1}}^{-1}\left[\int _ { 0 } ^ { t _ { 1 } } \operatorname { e x p } \left[i \int _ { T } M [ \varepsilon ( \tau ) ] \left(\xi_{1} \chi\left(\tau, t_{1}, \tau\right)+\right.\right.\right. \\
& \left.+\xi_{\chi}(0, t, \tau)\right) d \tau-\frac{1}{2} \int_{T T} b\left(\tau_{1}, \tau_{2}\right)\left(\xi_{1} \chi\left(\tau, t_{1}, \tau_{1}\right)+\xi_{\chi}\left(0, t, \tau_{1}\right)\right)\left(\xi_{1} \chi\left(\tau, t_{1}, \tau_{2}\right)+\right. \\
& \left.\left.\left.\left.+\xi \chi\left(0, t, \tau_{2}\right)\right) d \tau_{1} d \tau_{2}\right] d \tau\right]\left(x_{1}\right)\right](x)+f_{1}(x)^{*} F_{\xi}^{-1}\left[\int _ { 0 } ^ { t } \left(T_{B 0}\left(x_{1}\right)^{*}\right.\right. \\
& { }^{*} F_{\xi_{1}}^{-1}\left[\operatorname { e x p } \left[i \int_{T} M[\varepsilon(\tau)]\left(\xi_{1} \chi\left(0, t_{1}, \tau\right)+\xi \chi(\eta, t, \tau)\right) d \tau-\frac{1}{2} \iint_{T T} b\left(\tau_{1}, \tau_{2}\right) \times\right.\right. \\
& \left.\left.\times\left(\xi_{1} \chi\left(0, t_{1}, \tau_{1}\right)+\xi_{\chi}\left(\eta, t, \tau_{1}\right)\right)\left(\xi_{1} \chi\left(0, t_{1}, \tau_{2}\right)+\xi_{\chi}\left(\eta, t, \tau_{2}\right)\right) d \tau_{1} d \tau_{2}\right]\right]\left(x_{1}\right)+ \\
& +f_{1}\left(x_{1}\right)^{x_{1}} F_{\xi_{1}}^{-1}\left[\int _ { 0 } ^ { t _ { 1 } } \operatorname { e x p } \left[i \int_{T} M[\varepsilon(\tau)]\left(\xi_{1} \chi\left(\xi, t_{1}, \tau\right)+\xi \chi(\eta, t, \tau)\right) d \tau-\right.\right. \\
& -\frac{1}{2} \int_{T T} \int_{T} b\left(\tau_{1}, \tau_{2}\right)\left(\xi_{1} \chi\left(\xi, t_{1}, \tau_{1}\right)+\xi \chi\left(\eta, t, \tau_{1}\right)\right)\left(\xi_{1} \chi\left(\xi, t_{1}, \tau_{2}\right)+\right. \\
& \left.\left.\left.\left.\left.+\xi_{\chi}\left(\eta, t, \tau_{2}\right)\right) d \tau_{1} d \tau_{2}\right] d \tau\right]\left(x_{1}\right)\right) d \eta\right](x)= \\
& ={\frac{T_{B 0}(x) T_{B 0}\left(x_{1}\right)}{\sqrt{\operatorname{det} G\left(0, t, 0, t_{1}\right)}}}^{\overrightarrow{\mathbf{x}}} \exp \left[-\frac{1}{8} G^{-1}\left(0, t, 0, t_{1}\right)\left(\begin{array}{c}
x-A(0, t) \\
x_{1}-A\left(0, t_{1}\right)
\end{array}\right),\left(\begin{array}{c}
x-A(0, t) \\
x_{1}-A\left(0, t_{1}\right)
\end{array}\right)\right]+ \\
& +\int_{0}^{t_{1}}{\frac{T_{B 0}(x) f_{1}\left(x_{1}\right)}{\sqrt{\operatorname{det} G\left(\tau, t_{1}, 0, t\right)}}}^{\overrightarrow{\mathbf{x}}} \exp \left[-\frac{1}{8} G^{-1}\left(\tau, t_{1}, 0, t\right)\left(\begin{array}{c}
x-A(0, t) \\
x_{1}-A\left(\tau, t_{1}\right)
\end{array}\right),\left(\begin{array}{c}
x-A(0, t) \\
x_{1}-A\left(\tau, t_{1}\right)
\end{array}\right)\right]+
\end{aligned}
$$




$$
\begin{aligned}
& +\int_{0}^{t}{\frac{T_{B 0}\left(x_{1}\right) f_{1}(x)}{\sqrt{\operatorname{det} G\left(0, t_{1}, \eta, t\right)}}}^{\stackrel{\overrightarrow{\mathbf{x}}}{*}} \exp \left[-\frac{1}{8} G^{-1}\left(0, t_{1}, \eta, t\right)\left(\begin{array}{c}
x-A\left(0, t_{1}\right) \\
x_{1}-A(\eta, t)
\end{array}\right),\left(\begin{array}{c}
x-A\left(0, t_{1}\right) \\
x_{1}-A(\eta, t)
\end{array}\right)\right]+ \\
& +\int_{0}^{t} d \xi \int_{0}^{t_{1}} \frac{f_{1}\left(x_{1}\right) f_{1}(x)}{\sqrt{\operatorname{det} G\left(\xi, t_{1}, \eta, t\right)}} * \operatorname{\vec {x}} \exp \left[-\frac{1}{8} G^{-1}\left(\xi, t_{1}, \eta, t\right)\left(\begin{array}{c}
x-A\left(\xi, t_{1}\right) \\
x_{1}-A(\eta, t)
\end{array}\right),\left(\begin{array}{c}
x-A\left(\xi, t_{1}\right) \\
x_{1}-A(\eta, t)
\end{array}\right)\right] .
\end{aligned}
$$

Используя свойства функции $\chi$ и взяв обратное преобразование Фурье [15] по $\xi$, получим (33).

Теорема 9. Пусть выполняются условия теорем 8 и 9, тогда дисперсионная функиия имеет вид

$$
D\left[T_{B}(t, x)\right]=\left.M\left[T_{B}(t, x) T_{B}\left(t_{1}, x_{1}\right)\right]\right|_{t_{1}=t}-M^{2}\left[T_{B}(t, x)\right] .
$$

Доказательство. Подставив в формулу определения дисперсионной функции равенства (32) и (33), при этом положив в последнем выражении $t_{1}=t, x_{1}=x$, получим (34).

\section{7. Математическое ожидание и дисперсионная функция решения задач} (5), (6) в случае гауссова случайного процесса

Применив указанный выше подход к системе уравнений (5)-(6) с начальными условиями (8) в случае гауссова распределения случайного процесса, получим:

математическое ожидание массовой доли до начала момента конденсации водяного пара

$$
M[s(t, x)]=s_{0}(x) * \frac{x}{\sqrt{2 \pi B(0, t, 0, t)}} \exp \left(-\frac{(x-A(0, t))^{2}}{2 B(0, t, 0, t)}\right)(x)
$$

дисперсионную функцию массовой доли до начала момента конденсации водяного пара

$$
D[s(t, x)]=\left.M\left[s(t, x) s\left(t_{1}, x_{1}\right)\right]\right|_{x_{1}=t}-M^{2}[s(t, x)] ; ;
$$

математическое ожидание массовой доли после начала конденсации

$$
\begin{aligned}
& M\left[s_{m}(t, x)\right]=s_{m 0}(x)^{*} \frac{1}{\sqrt{2 \pi B(0, t, 0, t)}} \exp \left(-\frac{(x-A(0, t))^{2}}{2 B(0, t, 0, t)}\right)+ \\
& +f\left(x, T_{B}\right)^{*} \int_{0}^{t} \frac{1}{\sqrt{2 \pi B(\tau, t, \tau, t)}} \exp \left(-\frac{(x-A(\tau, t))^{2}}{2 B(\tau, t, \tau, t)}\right) d \tau,
\end{aligned}
$$


дисперсионную функцию после начала конденсации

$$
D\left[s_{m}(t, x)\right]=\left.M\left[s_{m}(t, x) s_{m}\left(t_{1}, x_{1}\right)\right]\right|_{t_{1}=t}-M^{2}\left[s_{m}(t, x)\right] .
$$

Таким образом, в рамках модельных представлений могут быть рассчитаны математическое ожидание и дисперсионная функция массовой доли водяного пара.

\section{8. Степень влияния случайных факторов}

Оценим степень влияния случайных факторов на поведение системы, описываемой уравнениями (5)-(8). Для этого рассмотрим одномерный случай с уравнениями вида (2)-(4) с начальными условиями (8).

Легко проверить, что

$$
\begin{aligned}
& s_{d}(t, x)=s_{0}\left(x-\int_{0}^{t} u(s) d s\right) ; \\
& s_{m d}(t, x)=s_{m 0}\left(x-\int_{0}^{t} u(s) d s+\int_{0}^{t} f\left(T_{B}, x-\int_{s}^{t} u(\tau) d \tau\right) d s\right) ; \\
& T_{B d}(t, x)=T_{B 0}\left(x-\int_{0}^{t} u(s) d s+\int_{0}^{t} f_{1}\left(x-\int_{s}^{t} u(\tau) d \tau\right) d s\right)
\end{aligned}
$$

являются решениями соответствующих уравнений (2) - (4) с начальным условием (8). Тогда функции

$$
\begin{aligned}
& I_{s}(t, x)=\left|s_{d}(t, x)-M[s(t, x)]\right| ; I_{s_{m}}(t, x)=\mid s_{m d}(t, x)-M\left[s_{m}(t, x)\right] ; \\
& I_{T_{B}}(t, x)=\left|T_{B d}(t, x)-M\left[T_{B}(t, x)\right]\right|
\end{aligned}
$$

позволяют оценить погрешности решений при отказе от учета случайных факторов. Отметим, что при $B(a, b, c, d) \rightarrow 0$ выражения $\frac{1}{\sqrt{2 \pi B(a, b, c, d)}} \times$ $\times \exp \left(-\frac{(x-A(a, b))^{2}}{2 B(a, b, c, d)}\right)$ стремятся к $\delta$-функции Дирака, и $I(t, x) \rightarrow 0$. Таким образом, при $B(a, b, c, d) \rightarrow 0$ формулы (32), (35) и (36) «переходят» в формулы решения детерминированной задачи. Тем самым доказано, что если случайный процесс, отвечающий горизонтальной компоненте скорости ветра, обладает «быстроубывающей» функцией автокорреляции, то приближенное решение может быть получено посредством замены скорости ветра ее средним значением. Однако наблюдения за реальными атмосферными параметрами показывают, что, как правило, это условие невыполнимо. 
8.1. Пример. Для наглядной оценки влияния случайных факторов на поведение системы, описываемой уравнением (2), удобно рассмотреть следующий модельный пример.

С учетом рассмотренных выше результатов исследования автокорреляционной функции скорости ветра можно определить начальные условия и параметры случайного процесса, моделирующего скорость ветра, например, следующими соотношениями и значениями:

$$
s_{0}=\cos \frac{2 \pi}{L} x ; \quad u=2 ; \quad M[\varepsilon(\tau)]=2 ; \quad b\left(\tau_{1}, \tau_{2}\right)=\frac{1}{1+\left(\tau_{1}-\tau_{2}\right)^{2}} ;
$$

$T$ - модельное время.

Графическое представление решений уравнения (35) и детерминированного уравнения (37) с приведенными выше условиями дано на рис.3,4.

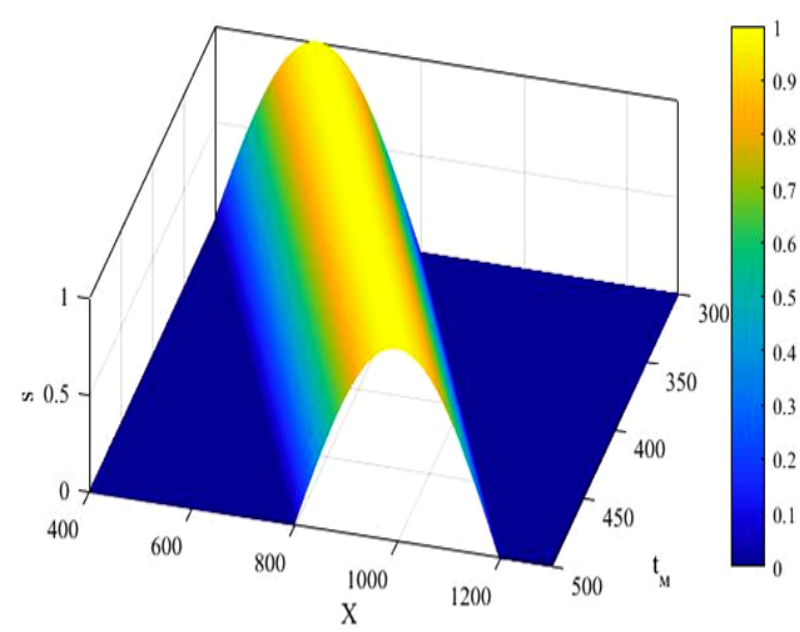

Рис.3. Решение детерминированного уравнения (37).

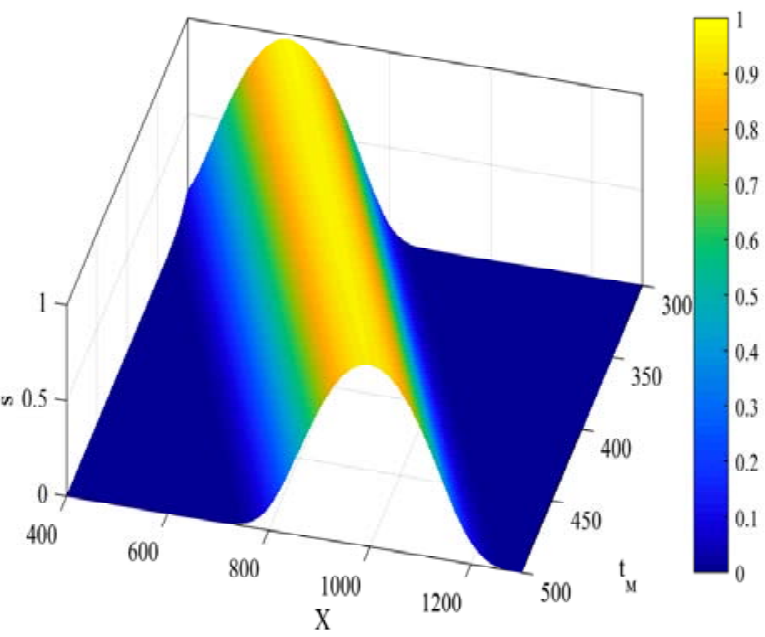

Рис.4. Решение выражения (35), учитывающее случайные (стохастические) факторы.

Из графиков видно, что множество решений детерминированной задачи на каждом шаге образует смещающееся в плоскости начальное условие, не изменяющееся с течением времени. В ситуации, когда скорость трактуется как случайный процесс (рис.4), математическое ожидание решения (35) распространяется со временем вдоль оси $x$ (условие нормализации предполагается выполненным).

Рассогласованность решений между математическим ожиданием стохастической задачи и детерминированной, рассмотрим в равномерной метрике $I_{s_{1}}(t)=\max _{x}\left|s_{d}(t, x)-M[s(t, x)]\right|$ и в смысле среднеквадратичного отклонения 
$I_{s_{2}}(t)=\sqrt{\int_{-\infty}^{+\infty}\left(s_{d}(t, x)-M[s(t, x)]\right)^{2} d x}$. Графически ее изменение показано на рис.5,6.

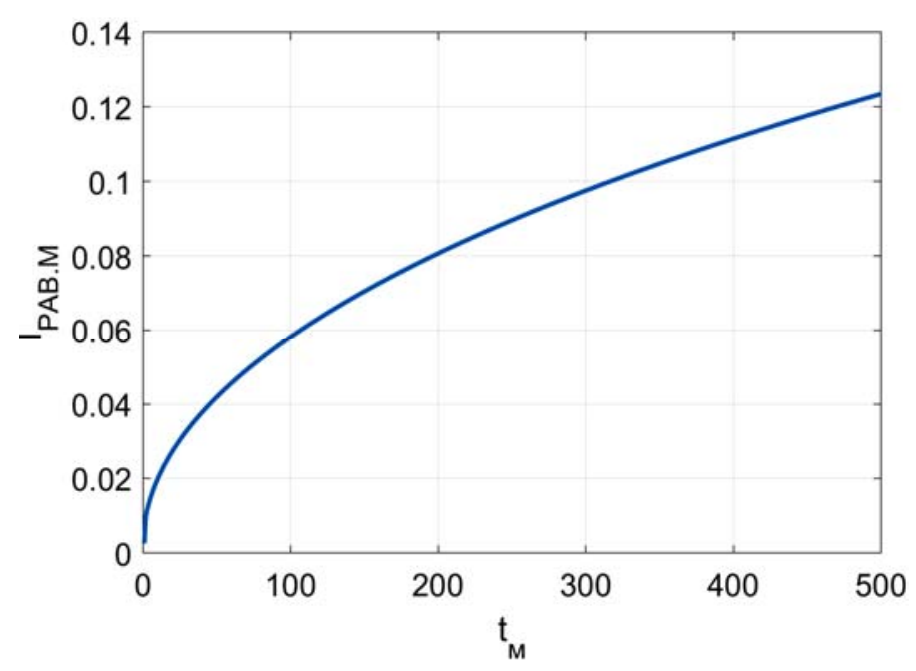

Pис.5. Разница между математическим ожиданием стохастической задачи и детерминированной в равномерной метрике.

Из рисунков видно, что обе функции монотонно растут с течением времени. Это говорит об увеличивающейся разнице между решением детерминированной задачи и математическим ожиданием решения соответствующего уравнения.

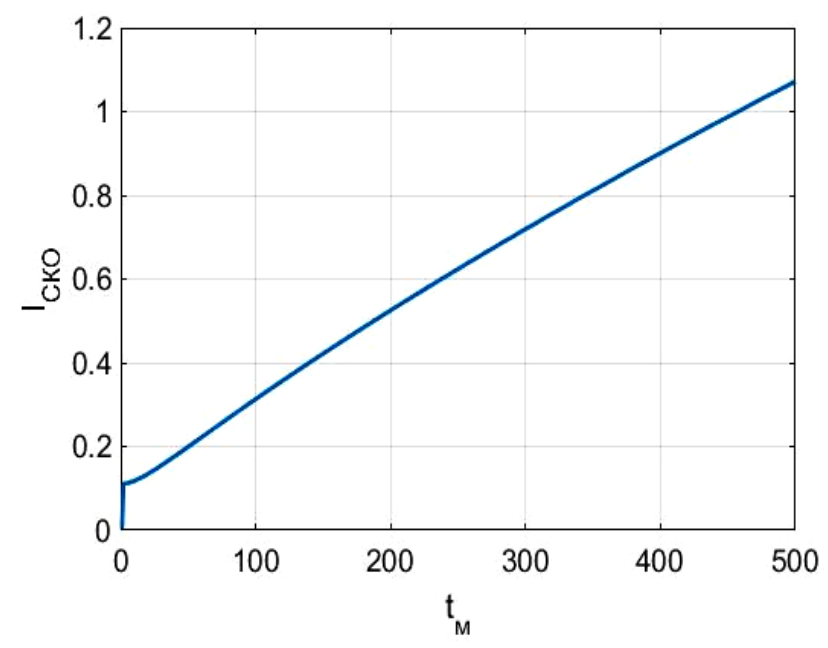

Рис.6. Разница между математическим ожиданием стохастической задачи и детерминированной в смысле среднеквадратического отклонения.

8.2. Эксперимент. Для верификации построенных моделей переноса тепла и влаги, позволяющих учитывать турбулентные свойства атмосферы, проведена серия экспериментов с использованием установки, в которую входили камера туманов, «аэродинамическая» труба и метеорологические датчики, измеряющие температуру и относительную влажность воздуха. Каме- 
ра туманов и «аэродинамическая» труба разделялись при помощи заслонки. В камере туманов создавались и регистрировались значения температуры и влажности воздуха, а также атмосферного давления. Затем порции воздуха с рассчитанной по известным соотношениям массовой долей водяного пара выпускались из камеры туманов в аэродинамическую трубу. Перенос массовой доли проходил за счет диффузии. Измерения температуры воздуха и относительной влажности проводились каждые 5 секунд вдоль всей трубы с шагом 0.5 м. В результате получены графики распределения массовой доли вдоль трубы. В качестве примера на рис.7 приведено распределения массовой доли, равной 0.002695 г/г, в аэродинамической трубе при штилевых условиях, температуре воздуха $20.7^{\circ} \mathrm{C}$, относительной влажности $94 \%$ и атмосферном давлении 1016.7 гПа.

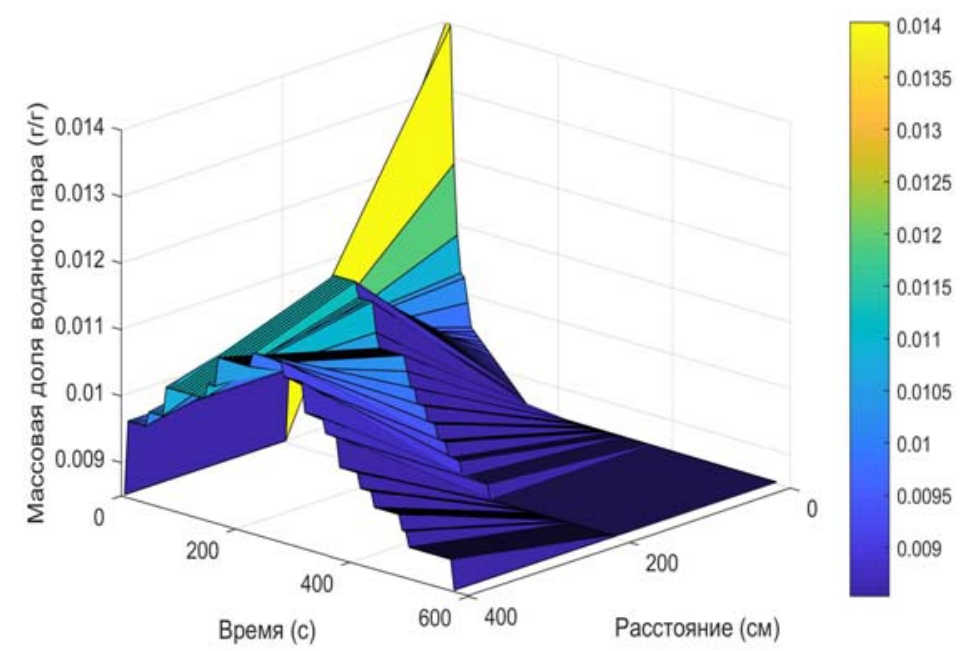

Рис.7. Экспериментальные данные о распределении массовой доли в «аэродинамической» трубе при штилевых условиях.

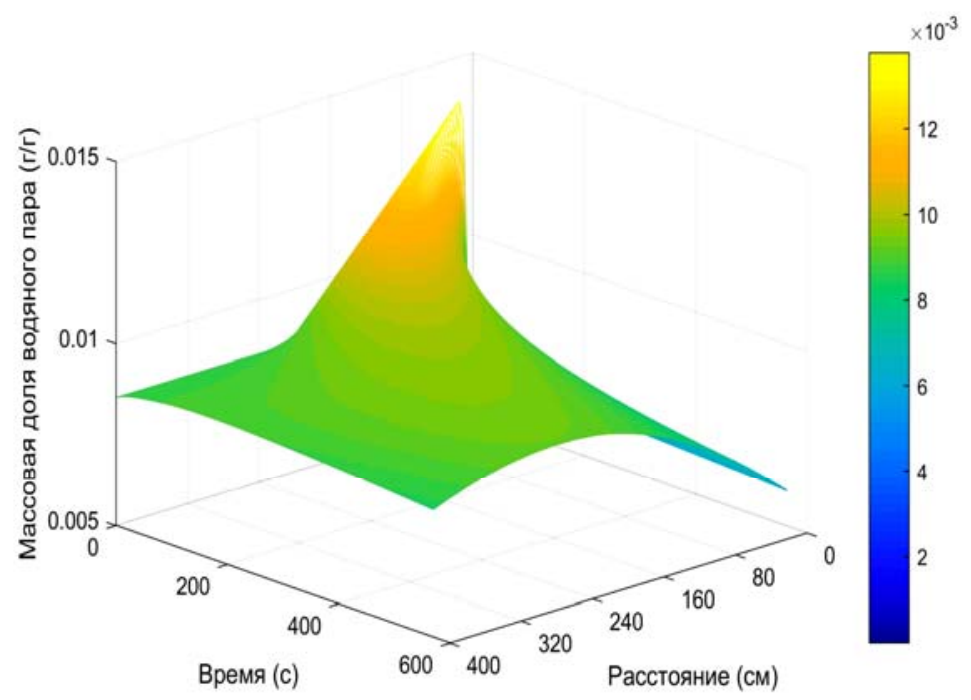

Рис.8. Эмпирическое распределение массовой доли в аэродинамической трубе при штилевых условиях. 
По результатам каждого испытания строилось теоретическое распределение массовой доли водяного пара. Для этого использовалось выражение (35), которое упростится, так как перенос происходил только за счет диффузии, и $M[\varepsilon(\tau)]=0$. При этом учитывалось начальное распределение массовой доли водяного пара в трубе. В результате были получены соответствующие графики, пример которых представлен на рис.8.

Рассогласованность решений также рассматривалась в равномерной метрике $I_{S_{\text {рав. }}}(t)=\max _{x}\left|s_{n p}(t, x)-M[s(t, x)]_{\text {теор }}\right|$ и в смысле среднеквадратичного отклонения $I_{S_{C K O}}(t)=\sqrt{\frac{1}{L} \int_{0}^{L}\left(s_{n p}(t, x)-M[s(t, x)]_{\text {mеор }}\right)^{2} d x}$, где $L-$ длина трубы (4 м). Соответствующие графики представлены на рис.9,10.

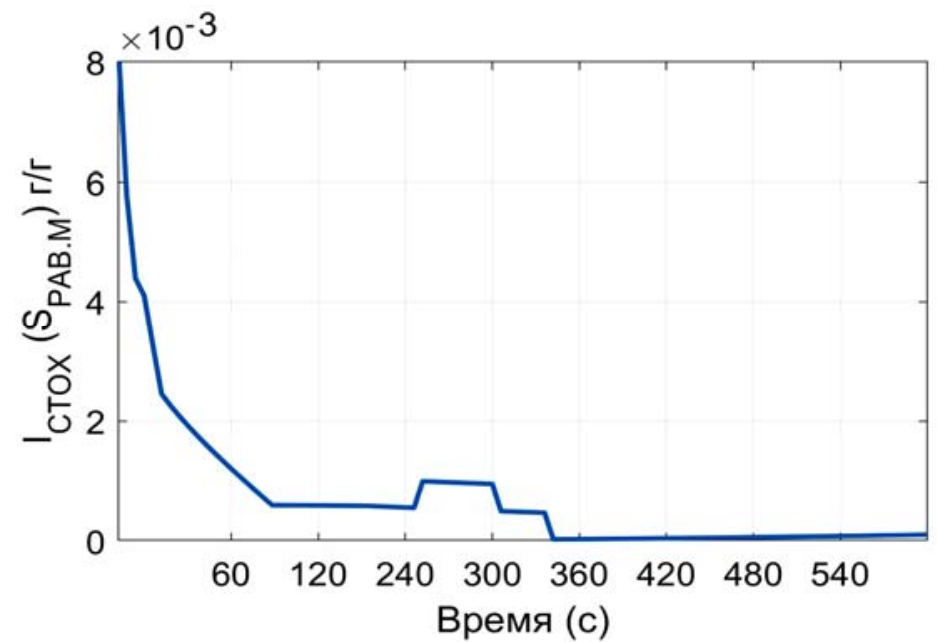

Рис.9. Рассогласованность практического и теоретического распределения в равномерной метрике.

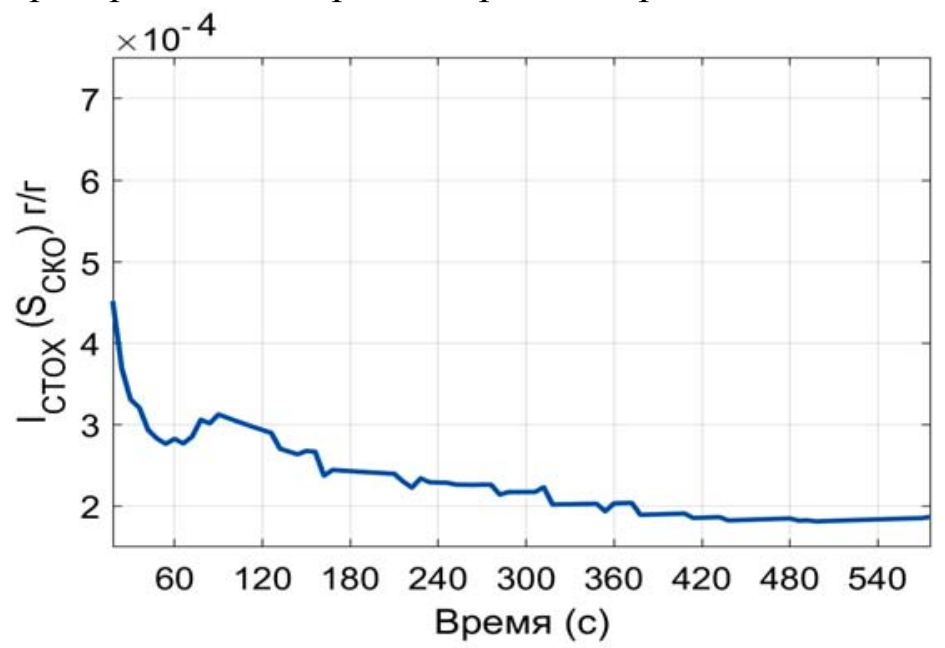

Рис.10. Рассогласованность практического и теоретического распределения в смысле среднеквадратичного отклонения. 
Из приведенных графиков видно, что с течением времени, ошибка между теоретическими и экспериментальными данными уменьшается. Это объясняется тем, что влагосодержание в трубе становится равным влагосодержанию в окружающей среде, которое в условиях отсутствия направленного переноса определяется флуктуациями движения воздушных масс и диффузией.

\section{9. Заключение}

В статье предложена новая модель переноса тепла и влаги в атмосфере, учитывающая влияние случайных неконтролируемых пульсаций скорости ветра, связанных с турбулентностью. В соответствующих уравнениях переноса проекции вектора скорости трактуются как случайные процессы с заданным характеристическим функционалом, который, в свою очередь, определяется по экспериментальным наблюдениям. Получены аналитические выражения для математического ожидания и дисперсионной функции решений дифференциальных уравнений в рамках сформулированного модельного представления, позволяющего учитывать турбулентные (стохастические) свойства атмосферы. Приведен пример для гауссова распределения. В рамках модельного примера показано, что не учет случайных факторов приводит к логарифмическому увеличению ошибки.

Возможные приложения предложенного в статье подхода применительно к метеорологическим задачам сосредоточены, прежде всего, в области прогнозирования погодных явлений малого пространственновременного масштаба (до нескольких километров и порядка нескольких часов). Именно в этих случаях учет случайных составляющих в моделях переноса представляется вполне оправданным, поскольку флуктуации скорости ветра могут вносить существенные изменения в распределения метеорологических величин. Предложенный подход может найти широкое применение, поскольку явление переноса часто рассматривается при совершенствовании различных технологических процессов.

\section{СПИСОК ЛИТЕРАТУРЫ}

1. M.L. Salby. Fundamentals of Atmospheric Physics. - NY.: Academic Press, 1996, 376 p.

2. M.A. Толстых. Глобальные модели атмосферы: современное состояние и перспективы развития // Труды ГНИЦ Российской Федерации, 2016, №359, с.5-32;

M.A. Tolstykh. Globalnye modeli atmosfery: sovremennoe sostoianie i perspektivy razvitiia // Trudy GNITS Rossiiskoi Federatsii, 2016, №359, s.5-32. 
3. Гидрометцентр России. http://old.meteoinfo.ru/faq. - Дата обращения: 13.05.2019. Gidromettsentr Rossii. http://old.meteoinfo.ru/faq. - Data obrashcheniia: 13.05.2019.

4. E.N. Lorenz. Deterministic nonperiodic flow // Journal of the atmospheric sciences, 1963, v.20. p.130-141.

5. А.Н. Багров. Оперативная численная схема прогноза конвективных явлений (кучевообразной облачности, ливней, гроз и шквалов) и обложных осадков // Труды ГМЦ, 1972, №91, с.29-38;

A.N. Bagrov. Operativnaia chislennaia skhema prognoza konvektivnykh iavlenii (kuchevoobraznoi oblachnosti, livnei, groz i shkvalov) i oblozhnykh osadkov // Trudy GMTS, 1972, №91, s.29-38.

6. Б.Д. Успенский. Количественный прогноз обложных и ливневых осадков // Метеорология и гидрология, 1970, №1, c.11-18;

B.D. Uspenskii. Kolichestvennyi prognoz oblozhnykh i livnevych osadkov // Meteorologiia i gidrologiia, 1970, №1, s.11-18.

7. В.Г. Задорожний. Стабилизация линейных систем мультипликативным случайным шумом // Дифференциальные уравнения, 2018, т.54, №6, с.734-753;

англ. пер.: V.G. Zadorozhniy. Stabilization of Linear Systems by a Multiplicative Random Noise // Differential Equations, 2018, v.54, №6, p.728-747.

8. В.Г. Задорожний. Методы вариационного анализа. - М.-Ижевск: РХД, 2006, 316 с.; V.G. Zadorozhnii. Metody variatsionnogo analiza. - M.-Izhevsk: RKHD, 2006, $316 \mathrm{~s}$.

9. В.Г. Задорожний. Линейный хаотический резонанс при вихревом движении // ЖВМ и МФ, 2013, т.53, №4, с.639-655;

англ. пер.: V.G. Zadorozhniy. Linear chaotic resonance in vortex motion // Comp. Math. \& Math. Phys., 2013, v.53, №4, p.486-502.

10. П.Г. Фрик. Турбулентность: Подходы и модели. - М.-Ижевск: ИКИ, 2003, 291 с.;

P.G. Friik. Turbulentnost: Podkhody i modeli. - M.-Igevsk: IKI, 2003, 291 s.

11. Б. Оксендаль. Стохастические дифференциальные уравнения. - М.: Мир, 2003, 408 с.;

B. Oksendal. Stochastic differential equations. - Berlin: Springer, 2003, 379 p.

12. А.Н. Колмогоров, С.В. Фомин. Элементы теории функций и функционального анализа - 6-е изд., исправл. - М.: Наука, 1989, 624 с.;

A.N. Kolmogorov, S.V. Fomin. Elementy teorii funktsii i funktsionalnogo analiza - 6-e izd., ispravl. - M.: Nauka, 1989, $624 \mathrm{~s}$.

13. Г.Е. Шилов. Математический анализ. Второй специальный курс. - М.: Наука, 1965, $328 \mathrm{c}$;

G.E. Shilov. Matematicheskii analiz. Vtoroi spetsialnyi kurs. - M.: Nauka, 1965, 328 s.

14. V.S. Nozhkin et al. Stochastic model of moisture motion in atmosphere // Journal of Physics: Conference Series, 2018, v.1096. 012167. doi:10.1088/1742-6596/1096/1/012167.

15. В.С. Владимиров. Обобщенные функции в математической физике. 2-е изд., испр. и доп. - М.: «Наука», 1979, 320 с.;

V.S. Vladimirov. Generalized functions in mathematical physics. - Mir Publishers, 1979, $390 \mathrm{p}$.

Поступила в редакцию 21.10.2019

После доработки 21.10.2019

Принята к публикации 25.11.2019 